\title{
Remodeling the matrix: doxycycline modulates tuberculosis immunopathology
}

\author{
Jason D. Simmons and Thomas R. Hawn \\ Department of Medicine, University of Washington School of Medicine, Seattle, Washington, USA.
}

Pulmonary cavitation is a hallmark of Mycobacterium tuberculosis (Mtb) infection that provides an immune-privileged niche for extracellular bacillary replication, which associates with increased transmission rates, drug resistance, and chronic lung dysfunction following antituberculous therapy (ATT). Inhibitors of matrix metalloproteinases (MMPs), which are induced by Mtb infection, have shown efficacy in preclinical models and improved microbiologic and immunopathologic outcomes. In this issue of the $J C l$, Hao Miow et al. performed a double-blind, randomized controlled trial exploring host-directed effects of the MMP inhibitor doxycycline versus placebo when added to standard ATT for pulmonary tuberculosis. Doxycycline treatment over two weeks durably modulated host blood transcription profiles, including the resolution of inflammatory gene programs. Reduced immunopathology markers in doxycycline-treated participants also included improved lung cavity volumes and lower MMP levels in blood and sputum. These findings provide mechanistic insight and momentum for using experimental medicine trials to develop host-directed therapies for tuberculosis.

\section{Mtb immunity - friend and foe?}

Tolerance becomes a crime when applied to evil.

$$
\text { -Thomas Mann }
$$

In Thomas Mann's epic 1924 novel The Magic Mountain, tuberculosis (TB) is at the center of a narrative structure that explores human creativity, compromise, and corruption. As it was in the 1800 s, TB remains a leading cause of death worldwide. In 2019, there were 1.4 million deaths from TB (1). Humans and Mycobacterium tuberculosis (Mtb) are connected by tens of thousands of years of coevolution and mutual tolerance required for survival. Among the quarter of the world's population who are currently or previously infected with Mtb, the vast majority have no symptoms and tolerate or clear the infection without overt detrimental consequence. However, for some, the initial tolerance of infection may progress insidiously and culminate in severe symptoms. Antimicrobial treatment of Mtb is highly effective, but is marred by the need for lengthy therapy, drug toxicities, drug resistance, and chronic lung damage, which can occur despite eradication of the bacillus.

Although defects in cell-mediated immunity and antitumor necrosis factor therapies increase susceptibility to TB (2), the host response to Mtb can also result in inappropriate tissue damage that worsens outcomes. Lung injury following TB commonly results in functional impairments that persist beyond treatment (3). A recent study of patients with HIV-TB coinfection who had the pulmonary function test, serial spirometry, found that $33 \%$ of

\section{Related Article: https://doi.org/10.1172/JCl141895}

Conflict of interest: The authors have declared that no conflict of interest exists.

patients had impaired lung function at 12 months (4). Cavitary lung disease, a hallmark of pulmonary TB infection, not only increases risk for functional impairment but also is associated with increased Mtb burden (5), transmission rates (6), and risk of emergent antimicrobial resistance (7). Host directed therapies (HDTs) that limit immunopathology may synergize with antimicrobials to reduce treatment duration and inflammatory sequelae and improve outcomes (8). With the exception of corticosteroids for TB meningitis, HDTs for $\mathrm{TB}$ remain an aspirational goal with unrealized potential.

Matrix metalloproteinases (MMPs) are a family of zinc-dependent proteases that contribute to tissue remodeling by degrading extracellular matrix, including collagen and proteoglycans. Mtb infection induces expression of multiple MMPs, including MMP-9 $(9,10)$, which plays a critical role in the recruitment of macrophages during early granuloma formation in animal models $(11,12)$. However, lung MMP expression is linked to pulmonary cavity formation in the rabbit TB model (13) and MMP inhibition reduces Mtb dissemination in mice (11). Deletion of MMP-9 resulted in host-protective effects in the zebrafish model, including reduced bacterial growth and improved survival (12). Although a clear immunopathologic (versus protective, ref. 14) role for MMPs is inconsistent across studies, these preclinical models provide clues that targeting MMPs may improve outcomes in humans (12). Sputum concentrations of MMP-1, -2, $-3,-8$, and -9 positively correlate with $\mathrm{TB}$ severity score and normalize with antituberculous therapy (ATT) in humans (15). In a recent randomized control trial in South Africa (16), lung function was monitored by spirometry in patients with TB treated with standard therapy and randomized to various candidate HDTs. These included CC-11050, which is a phosphodiesterase 4 inhibitor that reduces lung cavity volumes and tissue MMP levels while enhancing 


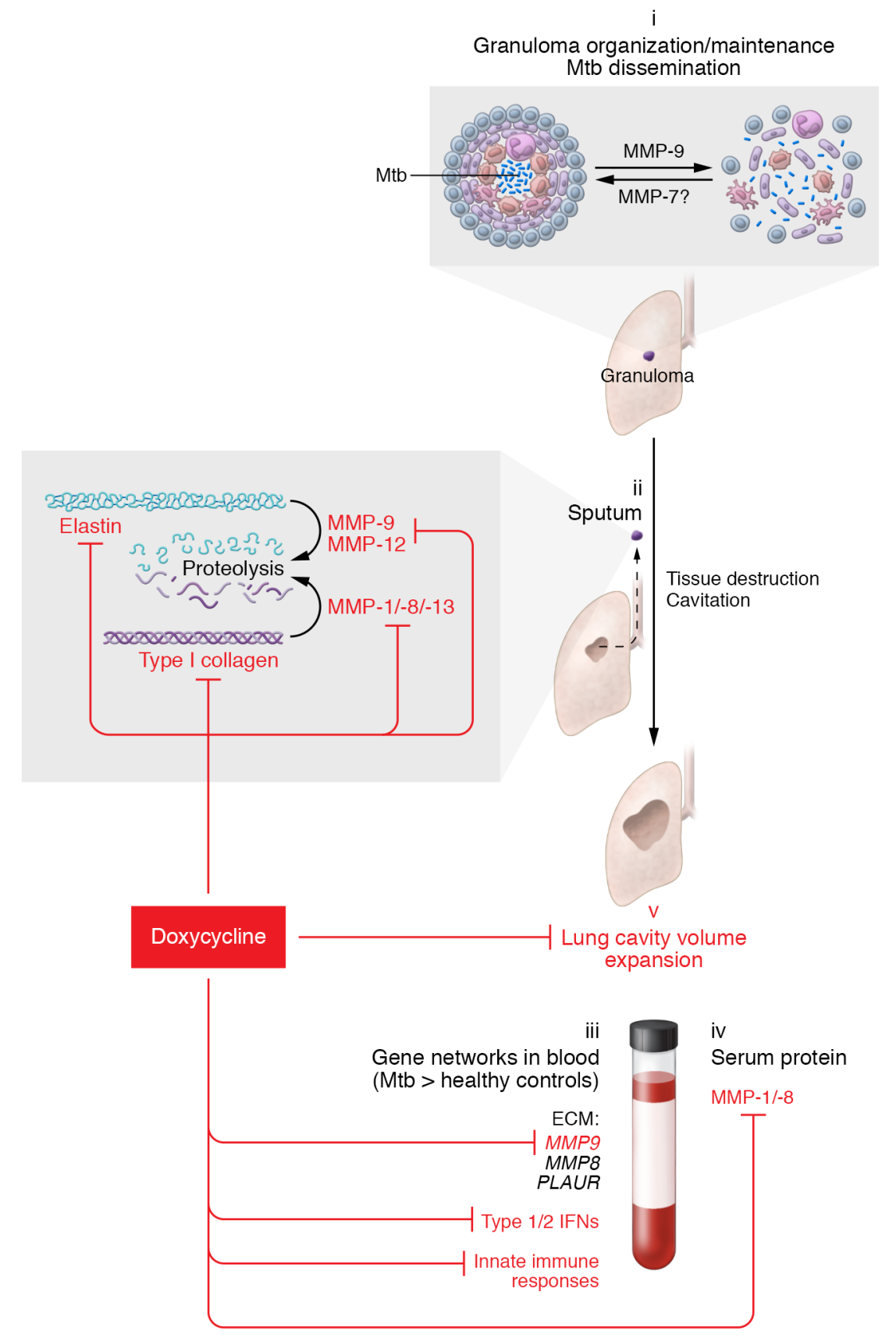

bacillary clearance in preclinical models (17). Patients randomized to CC- 11050 had significantly improved lung function after 180 days and a trend toward earlier culture clearance (16), suggesting that mitigation of lung damage also improves tissue delivery or otherwise enhances the efficacy of antimicrobials.

\section{Doxycycline modulates the host Mtb response}

Doxycycline is broadly recognized to have antiinflammatory properties, accounting for its efficacy in treating dermatologic diseases, such as bullous pemphigus (18) and acne, and granulomatous diseases, such as rosacea and sarcoidosis (19). Doxycycline is effective for these dermatologic diseases at subantimicrobial (less than 50 mg daily) doses that are also used to treat periodontal diseases where efficacy correlates with the inhibition of collagenase MMPs (20). Doxycycline has pleiotropic effects on host inflammatory cytokines, limits MMP-1 and MMP-3 secretion from Mtb-infected human monocyte-derived macrophages, and reduces collagenase activity of Mtb-infected neutrophil super-
Figure 1. The effect of doxycycline on pulmonary TB when added to standard therapy. (i) Aerosolized Mtb is initially contained within lung granulomas where it can remain quiescent or cause progressive pulmonary disease with unrestricted bacterial replication. (ii) In some cases, tissue destruction leads to pulmonary cavitation, which is associated with high mycobacterial burden and poor drug penetration, increasing the risk for transmission and emergent drug resistance. Mtb infection induces MMPs, which regulate inflammatory processes that range from tissue remodeling to tissue destruction (25) and are linked to granuloma maintenance and Mtb dissemination (10-12). MMPs also degrade elastin and collagen, contributing to cavitation (13). (iii) Hao Miow et al. explored the effects of doxycycline when added to standard antituberculous therapy in a randomized, placebo-controlled trial of patients with newly diagnosed pulmonary TB (23). Relative to healthy controls, multiple inflammatory gene programs were enriched among pulmonary TB subjects including coexpression modules related to extracellular matrix (ECM) genes. Longitudinal analysis of blood transcriptomes indicated doxycycline reduced transcription of MMPg and genes related to interferon (IFN) signaling and the innate immune response. These findings mirrored lower levels of MMP proteins in serum (iv) and and lower MMP-1, -8, $-9,-12,-13$ levels in sputum (ii). This correlated with decreased elastase (potentially including MMP-9 and -12) and collagenase (potentially including MMP-1, -8, -13) enzyme activities in sputum. While the study was underpowered to detect more relevant clinical or microbiologic outcomes, the broad MMP effects of doxycycline correlated with reduced lung cavity volumes (v) at two months, which is notable considering doxycycline was dosed only during the initial two weeks. natants (21). In respiratory epithelial cells, doxycycline inhibits MMP-1 promoter activity (22), suggesting it may modulate host transcription as opposed to direct inhibition of enzymatic function.

In this issue of the JCI, Hao Miow et al. report results on a randomized, double-blind, placebo-controlled trial measuring doxycycline host-directed activity in 30 patients with pulmonary TB (Figure 1) (23). The majority (63\%) of patients had pulmonary cavities and were positive for Mycobacteria in sputum tests (median Acid-Fast Bacillus 2+) at baseline. Doxycy- 
cline or placebo was added within 7 days of starting standard ATT and continued for 14 days only. Using coexpression network analysis of whole blood transcriptional profiles, gene clusters that specifically were downregulated with doxycycline treatment included the innate immune response, Type I and Type II interferon signaling. Transcripts from these gene clusters, including IRF1, APOL1, FCGR1A, and GBP6, which are upregulated by Mtb infection, remained elevated during ATT/ placebo treatment but were markedly downregulated in the ATT/doxycycline group at day 56. This occurred 42 days after completion of doxycycline and suggests either durable host-directed activity or pronounced early effects that shape the late immune response (23).

To explore early effects of doxycycline that may shape these later transcriptome changes, Hao Miow and colleagues next measured MMP transcript and protein levels in blood and sputum. After 14 days of treatment, decreased MMP9 transcripts and MMP-1 protein levels were measured in participants who received doxycycline, which were similar to the reduced MMP-1 and MMP-8 levels and collagenase activity measured in sputum. Importantly, the reduced MMP levels detected early following doxycycline treatment are unlikely to simply reflect nonspecific transcriptional effects, since they correlated tightly with measures of immunopathology. In the 26 patients who completed follow-up (13 in each arm), doxycycline treatment resulted in a notable reduction in pulmonary cavity volume (23). These doxycycline effects are consistent with other broad MMP inhibitors, such as marimastat and BB-94, that reduced pulmonary pathology in the murine model $(10,24)$. Together, these experimental medicine data suggest a potential benefit of doxycycline with a host pathway-dependent mechanism.

Degradation of extracellular matrix provides a niche for extracellular Mtb growth where ATT delivery is impaired (25), which may in part explain improved microbiologic outcomes following treatment with MMP inhibitors in preclinical pulmonary TB models $(10,22,24)$. However, a direct antimicrobial effect of doxycycline against Mtb cannot be ruled out. In broth culture, doxycycline was bacteriostatic (22) at concentrations (MIC 2.5 $\mu \mathrm{g} / \mathrm{mL}$ ) that are within peak serum levels measured previously after standard dosing (26). Doxycycline is used as second-line therapy for M. kansasii and M. fortuitum infections, and the related tetracycline minocycline is effective for M. leprae. No statistically significant microbiologic outcomes differed between groups in the trial by Hao Miow et al., although a trend toward lower median sputum bacterial titers was measured in participants who received doxycycline versus placebo (25 versus $100 \mathrm{CFU} / \mathrm{mL}, P=0.32)(23)$.

\section{Clinical implications}

While the durability of doxycycline effects on transcriptome profiles is promising, larger human trials with meaningful clinical endpoints, such as time to culture conversion and pulmonary function, are needed. These studies would ideally include participants with early cavitary disease and implement computerized tomography lung imaging on a subset to confirm doxycycline effects on cavity volumes, which were estimated by 2-dimensional radiographs in the current study. Furthermore, whether standard doxycycline dosing (100 mg twice daily) is optimal and whether treatment beyond 14 days results in greater benefits require further evaluation. In addition, the molecular target of doxycycline remains elusive. With over 24 MMP family members in mammals, doxycycline likely has pleiotropic effects and dissecting the breadth and potency of its inhibitory effects on all MMP family members, along with lung cell-specific expression patterns, could provide additional mechanistic insight $(19,25,27)$. Previous enzyme inhibition studies with MMPs purified from various tissues suggest high IC50 values in the micromolar range and raise questions about doxycycline target specificity (28-30), including possibilities of MMPindependent immunologic effects such as modulation of interferon pathways. Future studies could also delineate the cellular sources of inflammatory pathway changes measured in blood transcriptomes (e.g., with scRNAseq or Cite-seq analysis) and whether doxycycline primarily modulates myeloid cell populations or Mtb antigendependent $\mathrm{T}$ cell responses. Finally, there may also be non-MMP and immune-independent effects of doxycycline, including those on the host microbiome. Together, these data justify efforts to uncover immunologic specificity to identify targets for the development of host-directed therapies.

As a widely prescribed drug with an excellent tolerability and safety profile, doxycycline holds great promise as an HDT that could easily be implemented in clinical practice. Hao Miow et al. (23) demonstrate the powerful insights that can be generated with RCTs and an experimental medicine question. Pharmacologically targeting the host creates opportunities and approaches to TB therapy. Perhaps such strategies can overcome the common tolerance response of humans toward Mtb, which is too often associated with delayed antimicrobial effects, immunopathology, and poor outcomes. After thousands of years of coevolutionary tolerance between humans and Mtb, creative and precise harnessing of human immune responses are likely needed to both activate sterilizing responses and mitigate pathologic effects.

Address correspondence to: Thomas R. Hawn, University of Washington, 1959 NE Pacific Street, Seattle, Washington 98115, USA. Phone: 206.616.4124; Email: thawn@uw.edu.

1. World Health Organization. Global Tuberculosis Report. 2019. https://apps.who.int/iris/bitstream/ handle/10665/329368/9789241565714-eng.pdf. Accessed July 6, 2021.

2. Keane J, et al. Tuberculosis associated with infliximab, a tumor necrosis factor alpha-neutralizing agent. $N$ Engl J Med. 2001;345(15):1098-1104.

3. Pasipanodya JG, et al. Pulmonary impairment after tuberculosis. Chest. 2007;131(6):1817-1824.

4. Auld SC, et al. Pulmonary restriction predicts long-term pulmonary impairment in people with HIV and tuberculosis. BMC Pulm Med. 2021;21(1):19.

5. Urbanowski ME, et al. Cavitary tuberculosis: the gateway of disease transmission. Lancet Infect Dis. 2020;20(6):e117-e128.

6. Golub JE, et al. Delayed tuberculosis diagnosis and tuberculosis transmission. Int J Tuberc Lung Dis. 2006;10(1):24-30.

7. Kempker RR, et al. Additional drug resistance in Mycobacterium tuberculosis isolates from resected cavities among patients with multidrug-resistant or extensively drug-resistant pulmonary tuberculosis. Clin Infect Dis. 2012;54(6):e51-e54.

8. Hawn TR, et al. Host-directed therapeutics for tuberculosis: can we harness the host? Microbiol Mol Biol Rev. 2013;77(4):608-627.

9. Hrabec E, et al. Circulation level of matrix metalloproteinase-9 is correlated with disease severity in tuberculosis patients. Int J Tuberc Lung Dis. 2002;6(8):713-719. 
10. Izzo AA, et al. A matrix metalloproteinase inhibitor promotes granuloma formation during the early phase of Mycobacterium tuberculosis pulmonary infection. Tuberculosis (Edinb). 2004;84(6):387-396.

11. Taylor JL, et al. Role for matrix metalloproteinase 9 in granuloma formation during pulmonary Mycobacterium tuberculosis infection. Infect Immun. 2006;74(11):6135-6144.

12. Volkman HE, et al. Tuberculous granuloma induction via interaction of a bacterial secreted protein with host epithelium. Science. 2010;327(5964):466-469.

13. Kubler A, et al. Mycobacterium tuberculosis dysregulates MMP/TIMP balance to drive rapid cavitation and unrestrained bacterial proliferation. J Pathol. 2015;235(3):431-444.

14. Ordonez AA, et al. Matrix metalloproteinase inhibition in a murine model of cavitary tuberculosis paradoxically worsens pathology. J Infect Dis. 2019;219(4):633-636.

15. Ugarte-Gil CA, et al. Induced sputum MMP-1, -3 \& -8 concentrations during treatment of tuberculosis. PLoS One. 2013;8(4):e61333.

16. Wallis RS, et al. Adjunctive host-directed therapies for pulmonary tuberculosis: a prospective, open-label, phase 2, randomised controlled trial [published online March 16, 2021]. Lancet Respir Med. https://doi.org/10.1016/S22132600(20)30448-3.

17. Subbian S, et al. Adjunctive phosphodiesterase-4 inhibitor therapy improves antibiotic response to pulmonary tuberculosis in a rabbit model. EBioMedicine. 2016;4:104-114.

18. Williams HC, et al. Doxycycline versus prednisolone as an initial treatment strategy for bullous pemphigoid: a pragmatic, non-inferiority, randomised controlled trial. Lancet. 2017;389(10079):1630-1638.

19. Monk E, et al. Clinical applications of non-antimicrobial tetracyclines in dermatology. Pharmacol Res. 2011;63(2):130-145.

20. Golub LM, et al. Adjunctive treatment with subantimicrobial doses of doxycycline: effects on gingival fluid collagenase activity and attachment loss in adult periodontitis. JClin Periodontol. 2001;28(2):146-156.

21. Ong CW, et al. Neutrophil-derived MMP-8 drives AMPK-dependent matrix destruction in human pulmonary tuberculosis. PLoS Pathog. 2015;11(5):e1004917.

22. Walker NF, et al. Doxycycline and HIV infection suppress tuberculosis-induced matrix metalloproteinases. Am J Respir Crit Care Med.
2012;185(9):989-997.

23. Hao Miow Q, et al. Doxycycline host-directed therapy in human pulmonary tuberculosis. J Clin Invest. 2021;131(15):e141895.

24. Xu Y, et al. Matrix metalloproteinase inhibitors enhance the efficacy of frontline drugs against Mycobacterium tuberculosis. PLoS Pathog. 2018;14(4):e1006974.

25. Ong CW, et al. Tuberculosis, pulmonary cavitation, and matrix metalloproteinases. Am J Respir Crit Care Med. 2014;190(1):9-18.

26. Holmes NE, Charles PGP. Safety and efficacy review of doxycycline. Clin Med Ther. 2009;(1):471-482.

27. Vandenbroucke RE, Libert C. Is there new hope for therapeutic matrix metalloproteinase inhibition? Nat Rev Drug Discov. 2014;13(12):904-927.

28. Golub LM, et al. Tetracyclines inhibit connective tissue breakdown by multiple non-antimicrobial mechanisms. Adv Dent Res. 1998;12(2):12-26.

29. Golub LM, et al. Doxycycline inhibits neutrophil (PMN)-type matrix metalloproteinases in human adult periodontitis gingiva. J Clin Periodontol. 1995;22(2):100-109.

30. Smith GN Jr., et al. Specificity of inhibition of matrix metalloproteinase activity by doxycycline: relationship to structure of the enzyme. Arthritis Rheum.1999;42(6):1140-1146. 